\title{
Fractional-Order High-Pass Filter with Electronically Adjustable Parameters
}

\author{
Jan Jerabek, Roman Sotner, Jan Dvorak, Lukas Langhammer, Jaroslav Koton \\ Faculty of Electrical Engineering and Communication \\ Brno University of Technology \\ Brno, Czech Republic \\ jerabekj@feec.vutbr.cz
}

\begin{abstract}
This paper presents possible solution of fractional-order high-pass filter (FHPF) with electronically adjustable order between 1 and 2 and also with electronically adjustable pole frequency. It is based on well-known Follow-the-Leader Feedback (FLF) topology adjusted for utilization with operational transconductance amplifiers (OTAs) and adjustable current amplifiers (ACAs). This 3rd-order topology is used in order to approximate FHPF response in particular frequency band of interest. Design is supported by Pspice simulations for three particular values of the filter's order $(1.25,1.5,1.75)$ and for several values of pole frequency. Moreover, simulation results with two different approximations are compared.
\end{abstract}

Keywords- adjustable current amplifier; approximation; ACA; FHPF; FLF; follow-the-leader feedback; fractional-order; operational transconductance amplifier

\section{INTRODUCTION}

Traditionally, integer-order analog filters are studied [1]. Transition from pass band to stop band of these filters is based on the slope of integer-order approximation of the filter. Therefore, there is a step change of the order of the filter and their attenuation is equal to $20 n \mathrm{~dB} /$ decade theoretically, where $n$ is nonzero unsigned integer representing the order of the filter. However, synthesis of fractional-order electronic systems, for instance passive components, frequency filters or oscillators is of great interest of many research groups [1]-[19].

In case of the fractional-order filters, the change of the order of the filter is fractional, represented by $20 \cdot(n+a) \mathrm{dB} /$ decade slope of attenuation [1] in case of the low-pass (LP) or high-pass (HP) response and parameter $a$ follows this inequality: $0<a<1$.

Two common ways are used to obtain fractionalorder filter. The first is to use so-called fractionalorder element (FOE) in the filtering structure, because of absence of its physical form, usually approximated by passive RC network, e.g. [4]-[14]. Some filters are designed with respect to the passive prototype [8], $[10],[11],[14]$, some of them are based on the

Research described in this paper was financed by Czech Ministry of Education in frame of National Sustainability Program under grant LO1401 and by Czech Science Foundation projects under No. 14-24186P. For research, infrastructure of the SIX Center was used.
Kerwin-Huelsman-Newcomb (KHN) topology [9], [11]-[13] or Sallen-Key topology [9], [11] and usually including two FOEs [11]-[14]. Key disadvantage of FOE is that it is designed to behave as fractional element of particular order only in certain frequency band of interest, usually not exceeding two decades. For instance, it is band from 0.1 to $10 \mathrm{kHz}$ [10] or 0.2 to $20 \mathrm{kHz}$ [14].

There is a second way how to obtain fractionalorder filter. Fractional-order transfer function can be approximated by integer-order function of higher order [15]-[18]. Actually, the term $\boldsymbol{s}^{a}$ in the transfer function is approximated by a ratio of two rational polynomials (at least of second order). Its issue is similar to FOEs, it behaves as fractional element of particular order only in certain frequency. When third-order function is used, transfer function having fractional order between one and two can be obtained. It can be then implemented by various topologies, e.g. Follow the Leader Feedback (FLF) topology [15]-[18].

Standard non-tunable Operational Amplifier (OA) or Current Feedback Operational Amplifier (CFOA) are considered as active elements frequently [9-13], [17], but also advanced active elements, e.g. Differential Difference Current Conveyor (DDCC) [16], or electronically controllable elements, e.g. Operational Transconductance Amplifier and Adjustable Current Amplifier [18] are implemented in fractional-order filters. Design of low-pass (LP) filter with fractional character is presented frequently [8], [9], [11]-[13], [15]-[18], but there are also papers presenting solutions of high pass (HP) response [8], [9], [12], [13], [15] or band pass (BP) [9], [10], [12][14].

Some of journal papers provide great details about whole design procedure [15]. Note that pole frequency of reported filters is usually non-controllable and in range from $1 \mathrm{kHz}$ up to $10 \mathrm{kHz}$ [8]-[17], sometimes higher bands are tested [18].

Our contribution includes designed fractionalorder HP filter based on FLF with inverting integrators. Basic building blocks are Operational Transconductance Amplifiers (OTAs) [20] and singleinput Adjustable Current Amplifiers (ACAs) [21] active elements. The fractional order and pole frequency can be controlled electronically by control of the active element transfer coefficients. Design is supported by PSpice simulations with three different 
values of the fractional order $(1.25,1.5$ and 1.75) and for three particular values of pole frequency. Moreover, two common approximations are mutually compared in order to highlight their significant differences.

\section{BUTTERWORTH APPROXIMATION OF THE FRACTIONAL-ORDER HigH-PASS FILTER}

Design procedure of the fractional-order high-pass filter (FHPF) having order of $(1+a)$ was precisely described in [15]. Starting transfer function is in the following form:

$$
K_{1+a}^{H P}(s)=\frac{K_{1} s^{1+a}}{K_{2} s^{1+a}+K_{3} s+1},
$$

where from [15]:

$$
\begin{aligned}
& K_{1}=1, \\
& K_{2}=0.2937 a+0.71216, \\
& K_{3}=1.068 a^{2}+0.161 a+0.3324,
\end{aligned}
$$

coefficients $K_{\mathrm{X}}$, where $X=1,2,3$ influence directly the shape of the transfer characteristic. Note that the values of $K_{\mathrm{X}}$ are dependent on the parameter $a$, i.e. on the order of the filter. Second-order approximation of $\boldsymbol{s}^{a}$ from (1) is given by:

$$
\boldsymbol{s}^{a}=\frac{a_{0} \boldsymbol{s}^{2}+a_{1} \boldsymbol{s}+a_{2}}{a_{2} \boldsymbol{s}^{2}+a_{1} \boldsymbol{s}+a_{0}} .
$$

There are several ways how to approximate coefficients $a_{0}, a_{1}$ and $a_{2}$ from (3) and two of them are going to be compared within this paper. First set of coefficients can be calculated as follows [17]:

$$
\begin{aligned}
& a_{0}=2(1+a), \\
& a_{1}=5-a^{2}, \\
& a_{2}=2(1-a) .
\end{aligned}
$$

Second approximation [19] is defined by:

$$
\begin{aligned}
& a_{0}=a^{2}+3 a+2, \\
& a_{1}=8-2 a^{2}, \\
& a_{2}=a^{2}-3 a+2 .
\end{aligned}
$$

The following $3^{\text {rd }}$-order transfer function is going to be used to emulate FHPF with Butterworth approximation of order $(1+a)[15]$ :

$$
K_{1+a}^{H P}(\boldsymbol{s}) \cong \frac{K_{1}}{a_{0}} \frac{a_{0} \boldsymbol{s}^{3}+a_{1} \boldsymbol{s}^{2}+\boldsymbol{s} a_{2}}{\boldsymbol{s}^{3}+\frac{b_{1}}{b_{0}} \boldsymbol{s}^{2}+\frac{b_{2}}{b_{0}} \boldsymbol{s}+\frac{1}{b_{0}}} .
$$

Coefficients $b_{\mathrm{X}}$ are calculated directly form $a_{\mathrm{X}}$ and $K_{\mathrm{X}}$, where $X=1,2,3$, as shown below [15]:

$$
\begin{aligned}
& b_{0}=\frac{a_{0} K_{2}+a_{2} K_{3}}{a_{0}}, \\
& b_{1}=\frac{a_{1}\left(K_{2}+K_{3}\right)+a_{2}}{a_{0}}, \\
& b_{2}=\frac{a_{1}+a_{2} K_{3}+a_{2} K_{2}}{a_{0}} .
\end{aligned}
$$

One of possible ways how to implement $3^{\text {rd }}$-order transfer function is to use FLF filtering topology. Fig. 1 shows the particular block diagram implementing the topology, consisting of inverting integrators, supplemented by three feedback loops and three forward signal paths. Transfer function of this general topology is

$$
K(\boldsymbol{s})=\frac{\boldsymbol{I}_{O U T}}{\boldsymbol{I}_{I N}}=\frac{\boldsymbol{s}^{3} G_{1}+\boldsymbol{s}^{2} \frac{G_{2}}{\tau_{1}}+\boldsymbol{s} \frac{G_{3}}{\tau_{1} \tau_{2}}}{\boldsymbol{s}^{3}+\boldsymbol{s}^{2} \frac{1}{\tau_{1}}+\boldsymbol{s} \frac{1}{\tau_{1} \tau_{2}}+\frac{1}{\tau_{1} \tau_{2} \tau_{3}}}
$$

Equations how to obtain $\tau_{\mathrm{X}}$ and $G_{\mathrm{X}}$ from $a_{\mathrm{X}}$ and $b_{\mathrm{X}}$ coefficients are obvious when comparing (6) and (8), when $X=1,2,3$.

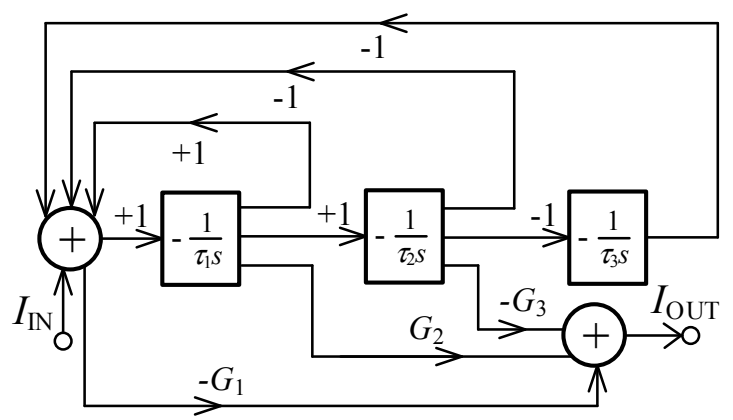

Figure 1. Block diagram of a 3rd-order FLF (follow-the-leader feedback) topology used for approximation of the FHPF (fractional-order high-pass filter) of $(1+a)$ order

\section{ACTIVE ELEMENTS USED IN FILTERING TOPOLOGY}

The designed filter, presented in the following section is based on two well-known types of active elements. First one is Operational Transconductance Amplifier (OTA) [20] with suitable number of current outputs. Schematic symbol of OTA in case of two current outputs is shown in Fig. 2. Note that this element is also frequently referred to as BalancedOutput OTA (BOTA).

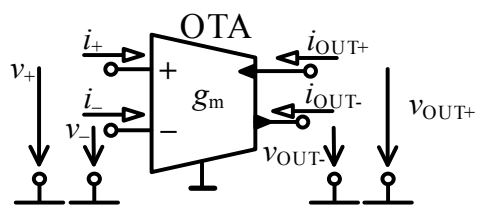

Figure 2. Operational Transconductance Amplifier (OTA) schematic symbol (dual-output version) 
Standard operation of OTA is described by these equations: $i_{\text {OUT }+}=-i_{\text {OUT }-}=g_{\mathrm{m}} \cdot\left(v_{+}-v_{-}\right)$, while $i_{+}=i_{-}=$ 0 . Note that transconductance parameter $\left(g_{\mathrm{m}}\right)$ of OTA is usually controlled by external DC current.

Second type of active element that is implemented in the proposed FLF topology of the filter is the singleinput Adjustable Current Amplifier (ACA) [21] with adjustable current gain. This element is depicted in Fig. 3. Number of current outputs also dependents on particular requirements.

Standard operation of ACA is described by simple equations: $i_{\mathrm{OUT}+}=-i_{\mathrm{OUT}-}=B \cdot i_{\mathrm{IN}}$. Current gain $(B)$ is also usually controlled externally, in many cases by DC voltage [22].

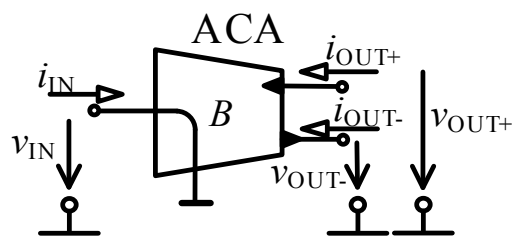

Figure 3. Adjustable Current Amplifier (ACA) schematic symbol (dual-output version)

Note that for current gain fixed to 1 or -1 , this element is frequently referred to as Current Follower (CF) in case of having single output. If there are two current outputs, element is known as Dual-Output $\mathrm{CF}$ (DO-CF) [23]. Description of DO-CF element is because of its simplicity omitted in this paper.

Utilization of OTA and ACA elements is beneficial because of the possibility of electronical control of parameters of the designed filter by electronic control of internal parameters of these active elements.

\section{Designed Fractional-ORder HP Filter}

Designed FHPF with active elements introduced in previous section and following the concept of FLF block topology (Fig. 1) is shown in Fig. 4.
Particular transfer function of the filter from Fig. 4 that has inverting character is as follows:

$$
K(\boldsymbol{s})=\frac{\boldsymbol{I}_{O U T}}{\boldsymbol{I}_{I N}}=\frac{N(\boldsymbol{s})}{D(\boldsymbol{s})},
$$

where

$$
N(\boldsymbol{s})=-\boldsymbol{s}^{3}-\boldsymbol{s}^{2} \frac{g_{m 1} B_{1}}{C_{1}}-\boldsymbol{s} \frac{g_{m 1} g_{m 2} B_{1} B_{2}}{C_{1} C_{2}},
$$

and

$$
\begin{aligned}
D(\boldsymbol{s}) & =\boldsymbol{s}^{3}+\boldsymbol{s}^{2} \frac{g_{m 1}}{C_{1}}+\boldsymbol{s} \frac{g_{m 1} g_{m 2} B_{1}}{C_{1} C_{2}}+ \\
& +\frac{g_{m 1} g_{m 2} g_{m 3} B_{1}}{C_{1} C_{2} C_{3}} .
\end{aligned}
$$

Fig. 4 contains all variables that are used in (9) and (10). Note that except values of $C_{1}, C_{2}$ and $C_{3}$, all these parameters can be controlled electronically, which is beneficial feature not only for control of the pole frequency of the filter but also for electronic change of the order of the HP filter as will be demonstrated in the following section.

\section{Simulation Results}

Filter from Fig. 4 was simulated in OrCAD PSpice under two scenarios in order to provide comparison of two possible approximations of $a_{0}, a_{1}$ and $a_{2}$ calculated from (4) and (5). All active elements were emulated by behavioral models as described below in order to provide realistic simulation results besides theoretical expectations. OTAs were substituted by $3^{\text {rd }}$-level model of Universal Current Conveyor (UCC) [23] and in case of ACAs, simulation model of EL2082 chip from Elantec [22] was used as a replacement. Unfortunately, EL2082 has only one current output, therefore, in case of DO-ACA1, additional copy of current is required. EL2082 was in this particular case supplemented by one UCC connected as DO-CF [24]. Note that DO-CF1 element within the structure shown in Fig. 4 was also emulated by UCC.

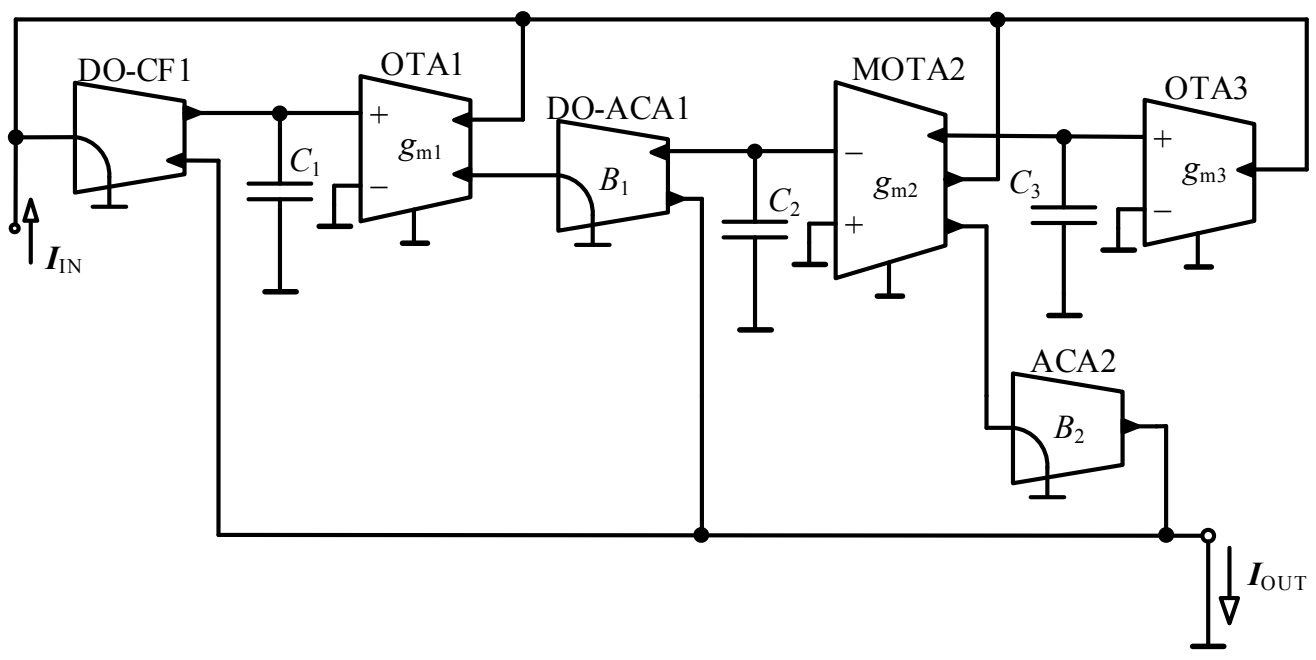

Figure 4. Designed $3^{\text {rd }}$-order HP filter approximating $(1+a)$-order FHPF with controllable active elements 
Starting pole frequency was selected as $f_{\mathrm{p}}=100$ $\mathrm{kHz}$, capacitor values are $C_{1}=560 \mathrm{pF}, C_{2}=910 \mathrm{pF}$ and $C_{3}=3.9 \mathrm{nF}$ for both approximations. Other calculated parameters of the filter are summarized for the first approximation in Table I and for the second approximation in Table II.

The simulation results in the case of the first values of coefficients (referred to as 1st set) and the second values coefficients (designated by 2nd set) respectively are compared to theoretical presumptions and also mutually in the following figures.

Fig. 5 provides comparison of theoretical expectations vs. theory for both approximations and for three values of $a$ parameter. From both graphs it is obvious that theory and simulation results match very well in case of first and also second approximation. Theoretically, attenuation in stop-band should be 25 , 30 and $35 \mathrm{~dB}$ per decade for chosen values of $a$ parameter. In case of first approximation, the obtained values are $24.1,29.3$ and $34.2 \mathrm{~dB}$, in case of second approximation obtained values are $25.5,30.5$ and 35.4 $\mathrm{dB}$.

In both cases particular band, where we can observe the validity of approximation is limited by the second-order approximation of $\boldsymbol{s}^{a}(3)$. Comparison of approximations in more detail is provided by Fig. 6 for both the magnitude and phase responses. As obvious from these graphs, second approximation has intended fractional character from $f_{\mathrm{p}}$ down to frequency around $4 \mathrm{kHz}$ (vs. $10 \mathrm{kHz}$ in the case of 1 st approximation). However the second approximation does not follow expected behavior of Butterworth approximation ideally in the pass band - there is visible attenuation around the pole frequency. Note that these findings are valid for all the tested values of $a$ parameter.

Based on these two key differences, proper type of approximation should be selected for particular application in order to obtain required character of response. Note that order of the FHPF is controlled electronically in both cases by simultaneous change of five parameters: $g_{\mathrm{m} 1}, g_{\mathrm{m} 2}, g_{\mathrm{m} 3}, B_{1}$, and $B_{2}$ as summarized in Table I and II. Moreover, if only $g_{\mathrm{m} 1}$, $g_{\mathrm{m} 2}, g_{\mathrm{m} 3}$ parameters are tuned, pole frequency can be controlled without disturbing the order of the filter as is shown in Fig. 7 for both approximations. Three $f_{\mathrm{p}}$-s were selected as an example: 33, 100 and $300 \mathrm{kHz}$. Change of $g_{\mathrm{m} 1}, g_{\mathrm{m} 2}, g_{\mathrm{m} 3}$ parameters required for setting of these pole frequencies is obvious from Table III, where values for both approximations are summarized. Note that comparison with theoretical expectations was omitted in this particular case.

TABLE I. PASSIVE COMPONENTS AND CURRENT GAINS FOR $\mathrm{A}=0.25,0.5,0.75$ WHEN FIRST SET OF COEFFICIENTS WAS USED (4)

\begin{tabular}{|c|c|c|c|}
\hline order $(\boldsymbol{a})$ & $\mathbf{0 . 2 5}$ & $\mathbf{0 . 5}$ & $\mathbf{0 . 7 5}$ \\
\hline $1 / g_{\mathrm{m} 1}[\Omega]$ & 988 & 1114 & 1157 \\
\hline $1 / g_{\mathrm{m} 2}[\Omega]$ & 1256 & 1179 & 978 \\
\hline $1 / g_{\mathrm{m} 3}[\Omega]$ & 1178 & 1040 & 1002 \\
\hline$B_{1}[-]$ & 0.686 & 0.621 & 0.516 \\
\hline$B_{2}[-]$ & 0.218 & 0.142 & 0.063 \\
\hline
\end{tabular}

TABLE II. PASSIVE COMPONENTS AND CURRENT GAINS FOR $\mathrm{A}=0.25,0.5,0.75$ WITH SECOND SET OF COEFFICIENTS (5)

\begin{tabular}{|c|c|c|c|}
\hline order $(\boldsymbol{a})$ & $\mathbf{0 . 2 5}$ & $\mathbf{0 . 5}$ & $\mathbf{0 . 7 5}$ \\
\hline $1 / g_{\mathrm{m} 1}[\Omega]$ & 722 & 863 & 980 \\
\hline $1 / g_{\mathrm{m} 2}[\Omega]$ & 1345 & 1220 & 983 \\
\hline $1 / g_{\mathrm{m} 3}[\Omega]$ & 1472 & 1164 & 1038 \\
\hline$B_{1}[-]$ & 0.712 & 0.607 & 0.493 \\
\hline$B_{2}[-]$ & 0.128 & 0.070 & 0.026 \\
\hline
\end{tabular}

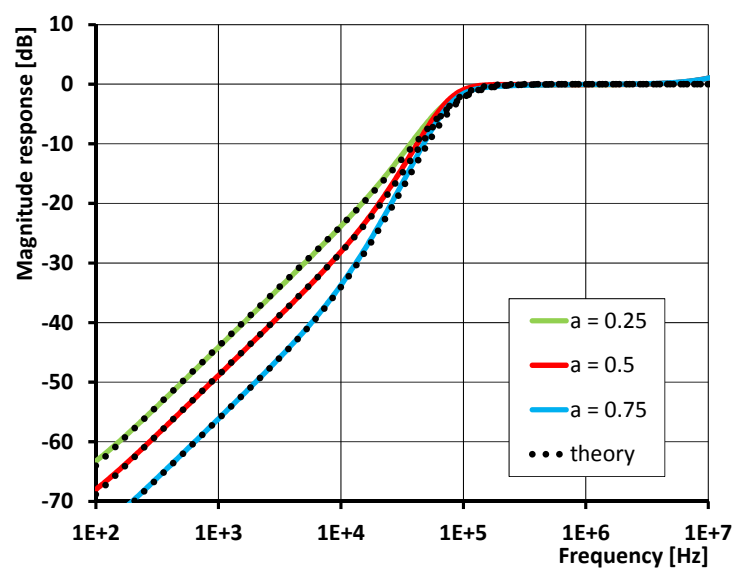

a)

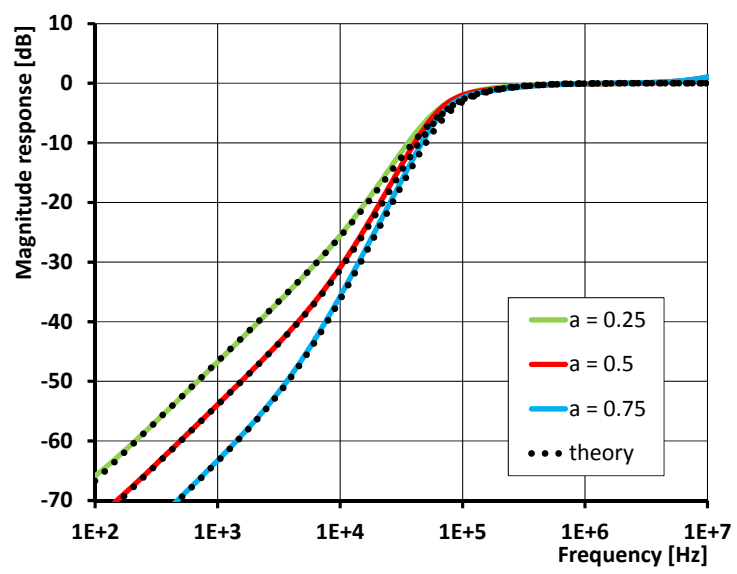

b)

Figure 5. Simulation results (colored solid lines) vs. theory (dotted lines) for three values of $a$ parameter (magnitude responses) in case of a) $1^{\text {st }}$ set of coefficients defined in (4), b) $2^{\text {nd }}$ set of coefficients defined by (5). Both for fixed value of $f_{\mathrm{p}}=100 \mathrm{kHz}$.

TABLE III. VALUES OF TRANSCONDUCTANCES WHEN TUNING THE POLE FREQUENCY IN BOTH THE DISCUSSED CASES AND FOR ORDER OF THE FILTER DEFINED BY A $=0.75$

\begin{tabular}{|c|c|c|c|}
\hline $\begin{array}{c}\text { Pole frequency } \boldsymbol{f}_{\mathbf{p}} \\
{[\mathbf{k H z}]}\end{array}$ & $\mathbf{3 3}$ & $\mathbf{1 0 0}$ & $\mathbf{3 0 0}$ \\
\hline $1 / g_{\mathrm{m} 1}\left(1^{\mathrm{st}}\right) / 1 / g_{\mathrm{m} 1}\left(2^{\text {nd }}\right)[\Omega]$ & $3471 / 2940$ & $1157 / 980$ & $386 / 327$ \\
\hline $1 / g_{\mathrm{m} 2}\left(1^{\mathrm{st}}\right) / 1 / g_{\mathrm{m} 2}\left(2^{\text {nd }}\right)[\Omega]$ & $2934 / 2949$ & $978 / 983$ & $326 / 328$ \\
\hline $1 / g_{\mathrm{m} 3}\left(1^{\mathrm{st}}\right) / 1 / g_{\mathrm{m} 3}\left(2^{\text {nd }}\right)[\Omega]$ & $3006 / 3114$ & $1002 / 1038$ & $334 / 346$ \\
\hline
\end{tabular}




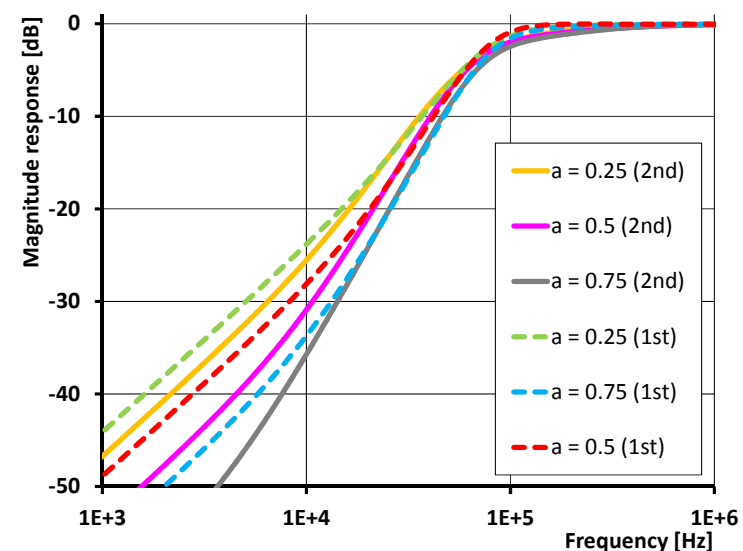

a)

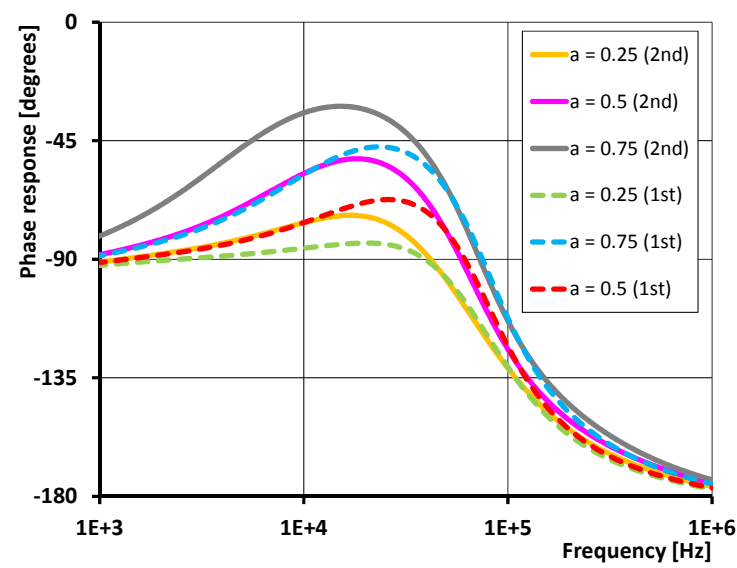

b)

Figure 6. Mutual comparison of both approximations in detail for three values of $a$ parameter and fixed value of $f_{\mathrm{p}}=100 \mathrm{kHz}$. a) magnitude responses b) phase responses.

From both magnitude and phase response graphs in Fig. 7 it is obvious that order of the filter is not disturbed while tuning the pole frequency in case of the simulation results. Moreover, these graphs confirm the previously observed differences between two studied approximations, i.e. that first approximation has more limited validity of fractional character in stop band, however, its features are very good in the pass band, because there is no undesired attenuation. Of course, these differences are given by particular positions of zeros and poles of transfer functions that are dependent on the selected type of approximation of transfer function.

Note that design calculation were aimed to allow omitting of ACAs, if a number of active elements should be decreased. Because for any value of $a$ parameter current gain is less than one $\left(B_{\mathrm{X}}<1\right)$. Therefore, if electronical control of order is not required, number of active elements can be decreased to only 4 active elements.

In case of this FHPF response, sufficiently high values of output resistances of used active elements are very important. In this particular case, it influences results significantly first below $30 \mathrm{~Hz}$ frequency and therefore it is not an issue in band where fractionalorder character is provided and it can be neglected.

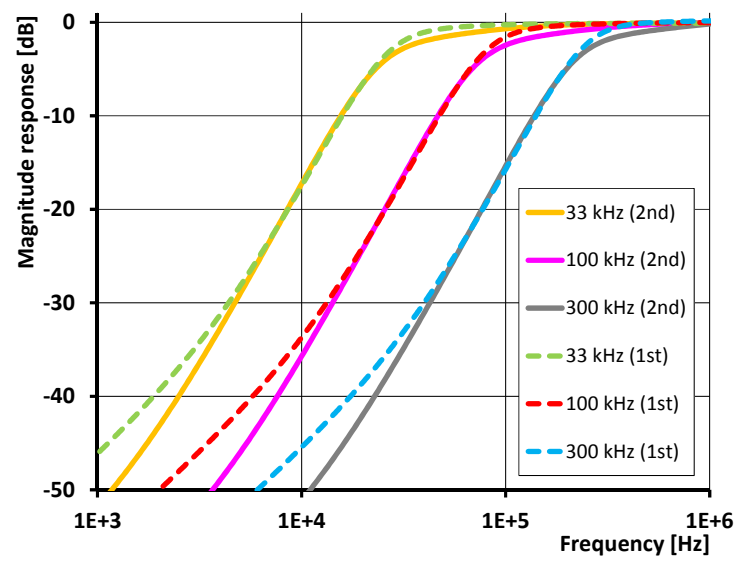

a)

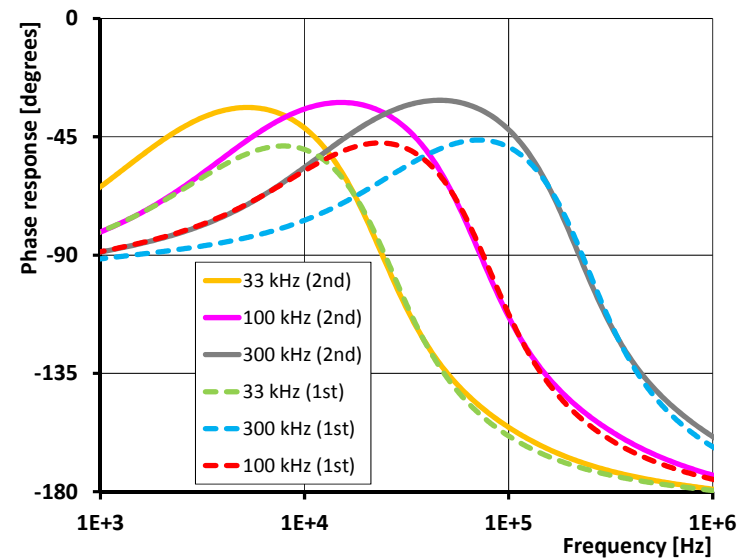

b)

Figure 7. Mutual comparison of both approximations in detail in case of tuning $f_{\mathrm{p}}$ and for $a=0.75 \mathrm{a}$ ) magnitude responses b) phase responses.

\section{CONCLUSION}

This paper presented design procedure and particular solution of fractional-order HP filter. The simulation results were obtained with help of behavioral models of OTAs and ACAs and the design is prepared in order to allow also future verification of features by laboratory measurements. Order of the filter and also the pole frequency of the filter can be controlled electronically. Moreover, two possible approximations were mutually compared in order to highlight their differences. As discussed in introductory part, most of the published solutions of fractional-order filters are designed to operate in lowfrequency range below $10 \mathrm{kHz}$ because of active elements used. The designed FHPF topology presented in this paper confirmed possibility to use concept of fractional-order filters also at higher frequencies. Of course, obtained features are always limited by features of active elements. Really precise values of transconductances, i.e. resistors, and also of current gains are required. Of course, in case of real implementation, the values of resistors have to be selected from at least E48 row, while precise values of current gains are usually not that problematic. This step was omitted in order to suppress impact of inaccurate resistors and to focus more on features of both approximations. 


\section{REFERENCES}

[1] A. Elwakil, "Fractional-order circuits and systems: An emerging interdisciplinary research area", IEEE Circuits and Systems Magazine, vol. 10, no. 4, pp. 40-50, Fourthquarter 2010

[2] B. T. Krishna, K.V.V.S. Reddy, "Active and Passive Realization of Fractance Device of Order 1/2", Active and Passive Electronic Components, Vol. 2008, pp. 1-5, 2008.

[3] M. C. Tripathy, D. Mondal, K. Biswas, S. Sen, "Experimental studies on realization of fractional inductors and fractionalorder bandpass filters", International Journal of Circuit Theory and Applications, vol. 43, no. 9, pp. 1183-1196, September 2015.

[4] K. Biswas, S. Sen, P. Dutta, "Realization of a constant phase element and its performance study in a differentiator circuit", IEEE Transactions on Circuits and Systems-II: Express Briefs, vol. 53, no. 9, pp. 802-806, September 2006.

[5] M. S. Krishna, S. Das, K. Biswas, B. Goswami, "Fabrication of a fractional order capacitor with desired specifications: A study on process identification and characterization", IEEE Transactions on Electron Devices, vol. 58, no.11, pp. 40674073, November 2011.

[6] D. Mondal, K. Biswas, "Performance study of fractional order integrator using single-component fractional order element", IET Circuits, Devices \& Systems, vol. 5, no. 4, pp. 334-342, July 2011.

[7] L. Dorcak , J. Valsa, E. Gonzalez, J. Terpak , I. Petras, L. Pivka, "Analogue Realization of Fractional-Order Dynamical Systems", Entropy, vol. 15, no. 10, pp. 4199-4214, October 2013.

[8] A. Radwan, A. Soliman, A. Elwakil, "First-order filters generalized to the fractional domain", Journal of Circuits, Systems, and Computers, vol. 17, no. 1, pp. 55-66, February 2008 .

[9] A. Radwan, A. Elwakil, A. Soliman, "On the generalization of second-order filters to the fractional-order domain", Journal of Circuits, Systems and Computers, vol. 18, no. 2, pp. 361-386, April 2009.

[10] P. Ahmadi, B. Maundy, A. Elwakil, L. Belostotski, "Highquality factor asymmetric-slope band-pass filters: a fractionalorder capacitor approach", IET Circuits, Devices and Systems, vol. 6, no.3, pp. 187-197, May 2012.

[11] A. Ali, A. Radwan, A. Soliman, "Fractional order Butterworth filter: active and passive realizations", IEEE Journal on Emerging and Selected Topics in Circuits and Systems, vol. 3, no. 3, pp. 346-354, September 2013.

[12] A. Soltan, A. Radwan, A. Soliman, "Fractional order filter with two fractional elements of dependant orders", Microelectronics Journal, vol. 43, no.11, pp. 818-827, November 2012.
[13] M. Tripathy, K. Biswas, S. Sen, "A design example of a fractional-order Kerwin-Huelsman-Newcomb biquad filter with two fractional capacitors of different order", Circuits Systems and Signal Processing, vol. 32, no. 4, pp 1523-1536, August 2013.

[14] M. C. Tripathy, D. Mondal, K. Biswas, S. Sen, "Experimental studies on realization of fractional inductors and fractional-order bandpass filters", International Journal of Circuit Theory and Applications, vol. 43, no. 9, pp. 11831196, September 2015.

[15] G. Tsirimokou, C. Laoudias, C. Psychalinos, “0.5-V fractional-order companding filters", International Journal of Circuit Theory and Applications, Vol. 43, No. 9, pp. 11051126, September 2015.

[16] F. Khateb, D. Kubanek, G. Tsirimokou, C. Psychalinos, "Fractional-order filters based on low-voltage DDCCs", Microelectronics Journal, in press, 2016.

[17] B. Maundy, A. Elwakil, T. Freeborn, "On the practical realization of higher-order filters with fractional stepping", Signal Processing, vol. 91, no. 3, pp. 484-491, March 2011.

[18] J. Jerabek, R. Sotner, D. Kubanek, J. Dvorak, L. Langhammer, N. Herencsar, K. Vrba, "Fractional-Order LowPass Filter with Electronically Adjustable Parameters", In Proceedings of the 39th International Conference on Telecommunications and Signal Processing - TSP 2016. Vienna, Austria, submitted.

[19] B. Maundy, A. Elwakil, T. Freeborn, "Field programmable analogue array implementation of fractional step filters", IET Circuits Devices and Systems, vol. 4, no. 6, pp. 514-524, 2010 .

[20] D. Biolek, R. Senani, V. Biolkova, Z. Kolka, "Active elements for analog signal processing: Classification, Review and New Proposals," Radioengineering, vol. 17, no. 4, pp. 1532, December 2008 .

[21] J. Jerabek, J. Koton, R. Sotner, K. Vrba, “Adjustable bandpass filter with current active elements: two fully-differential and single-ended solutions", Analog integrated circuits and signal processing, vol. 74, no. 1, pp. 129-139, January 2013.

[22] Intersil (Elantec). EL2082 CN Current-mode multiplier (datasheet), 1996, 14 p., accessible on www: http://www.intersil.com/data/fn/fn7152.pdf

[23] J. Jerabek, K. Vrba, "Multiple-Input Multiple- Output Universal Filter Using Current Followers", In Proceedings of the 31th International Conference on Telecommunications and Signal Processing - TSP 2008. Budapest, Hungary, pp. 29-32, 2008.

[24] R. Sotner, A. Kartci, J. Jerabek, N. Herencsar, T. Dostal, K. Vrba, "An Additional Approach to Model Current Followers and Amplifiers with Electronically Controllable Parameters from Commercially Available ICs", Measurement Science Review, vol. 12, no. 6, p. 255-265, 2012. 